\title{
Nonlinearities in Silicon Optical Fibers
}

\author{
P. Mehta ${ }^{1}$, N. Healy ${ }^{1}$, R. Slavík ${ }^{1}$, R.T. Watts ${ }^{3}$, J.R. Sparks ${ }^{2}$, T.D. Day ${ }^{2}$, P.J.A. Sazio ${ }^{1}$, \\ J.V. Badding ${ }^{2}$, and A.C. Peacock ${ }^{1}$ \\ ${ }^{1}$ Optoelectronics Research Centre, University of Southampton, Southampton SO17 1BJ, United Kingdom \\ ${ }^{2}$ Department of Chemistry and Materials Research Institute, Pennsylvania State University 16802 PA, USA \\ ${ }^{3}$ Research Institute for Networks and Communications Engineering, Dublin City University, Glasnevin, Dublin 9 , \\ Ireland \\ pm4g09@orc.soton.ac.uk
}

\begin{abstract}
The nonlinear propagation characteristics in a hydrogenated amorphous silicon core optical fiber are investigated to demonstrate ultra-fast all-optical switching by crossabsorption modulation.

(C) 2011 Optical Society of America

OCIS codes: (060.2270) Fiber characterization; (060.2290) Fiber materials; (060.4370) Nonlinear optics, fibers;
\end{abstract}

\section{Introduction}

Advances in semiconductor photonics are revolutionizing the future of modern optoelectronic devices. These devices have been of popular interest due to the low costs of mass fabrication associated with on-chip processing techniques. Recently, hydrogenated amorphous silicon $(\mathrm{a}-\mathrm{Si}: \mathrm{H})$ waveguides were differentiated from crystalline silicon due to their ease of fabrication and enhanced nonlinearity [1]. In addition, silicon contains a broad transmission window and strong light confinement, ideal for waveguiding. We use a high pressure chemical deposition technique to infiltrate silicon into nanometer to micrometer sized silica capillaries. This simple low-cost technique produces centimeter lengths of completely filled fibers of various micro-structured templates [2]. The integration of the active semiconductor material as the fiber core has the advantage of offering robustness and cylindrical geometry as well as providing the step towards an elementary unification of silicon photonics with existing fiber infrastructures.

In order to establish the feasibility of this novel waveguide for use in modern photonic systems, we experimentally and theoretically investigate pulse propagation characteristics in the presence of two-photon absorption (TPA), freecarrier absorption (FCA), and self-phase modulation (SPM) of a step index silicon fiber. The TPA parameter, FCA parameter and nonlinear refractive index are determined and found to be larger [3] than those of crystalline silicon, in agreement with measurements on a-Si:H on-chip waveguides. Finally, as the first step towards an all optical nonlinear device, we demonstrate an ultra-fast, ultra-compact amplitude modulator.

\section{Fabrication and fiber properties}

Deposition of amorphous silicon in a $6 \mu \mathrm{m}$ silica capillary was performed by flowing a mixture of silane and hydrogen $\left(\mathrm{SiH}_{4} / \mathrm{H}_{2}\right)$ through the central air core under high pressures ( $\left.\sim 35 \mathrm{MPa}\right)$ while simultaneously heating the fiber in a tube furnace. Over a low temperature range of $320-400^{\circ} \mathrm{C}$, a hydrogenated form of amorphous silicon accumulates within the central hole of the capillary assuming the pristine surface smoothness of the silica cladding. The incorporation of hydrogen is well known to passivate dangling bond defects in amorphous semiconductors dramatically reducing transmission losses [4]. Post-deposition procedures routinely involve material characterizations such as SEM images to identify the fill factor at nanometer resolution, and spontaneous Raman spectroscopy to confirm the presence of phonon modes associated with a-Si:H bonds.

A completely filled silicon-core fiber section of length $\sim 2 \mathrm{~cm}$ is studied. Due to the high index contrast between silicon and silica $(\Delta n \sim 2.1)$, modal analysis based on a finite element model for pulse propagation simulations were used to calculate the effective area of $A_{\text {eff }} \sim 14 \mu \mathrm{m}^{2}$ and the group velocity dispersion (GVD) of $\beta_{2} \sim 1 \mathrm{ps}^{2} / \mathrm{m}$ at $1540 \mathrm{~nm}$. The linear absorption coefficient for the fiber was experimentally measured at $1540 \mathrm{~nm}$ to be $\alpha_{l} \sim 1.7 \mathrm{~dB} / \mathrm{cm}$ marking the lowest recorded transmission loss for a-Si:H optical fibers.

\section{Theory and experiment}

Nonlinear absorption measurements are conducted using a $650 \mathrm{fs}$ high power fiber laser at a $40 \mathrm{MHz}$ repetition rate. The input power is varied and the output recorded and fitted to a simplified propagation equation describing the temporal 


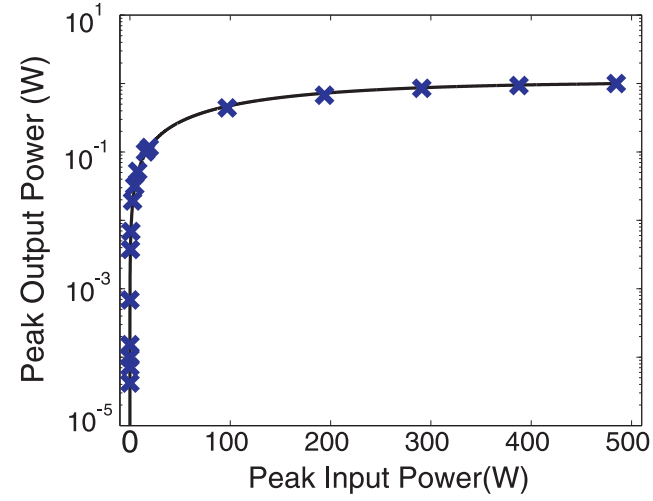

(a)

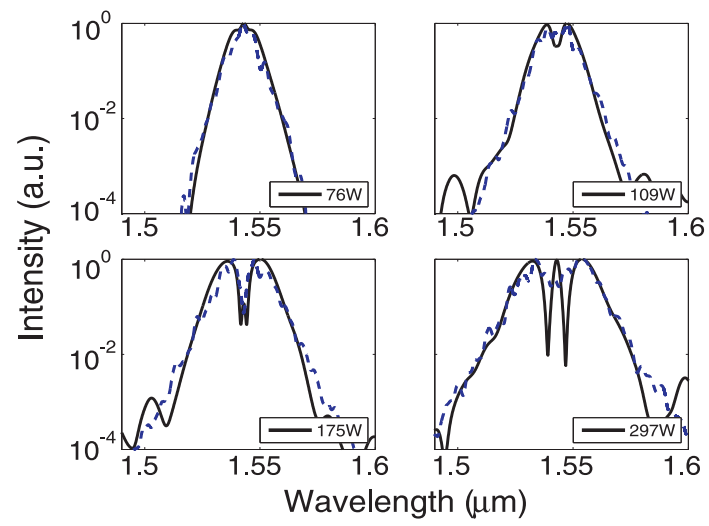

(b)

Fig. 1. (a) Nonlinear absorption measurements fitted (black curve) using Eqs. (1) and (2). (b) Evolution of self-phase modulation as a function of peak coupled input power. Solid black lines represent simulated fits using Eqs. (2) and (3). Broken lines are measured spectra.

evolution of the initial intensity profile $I(t, z)$ which is solved in conjunction with the free carrier density $N(t, z)$. The coupled equations can be expressed as [5]:

$$
\begin{gathered}
\frac{d I(t, z)}{d z}=-\alpha_{l} I(t, z)-\beta_{\mathrm{TPA}} I^{2}(t, z)-\sigma N(t, z) I(t, z), \\
\frac{d N(t, z)}{d t}=\frac{\beta_{\mathrm{TPA}}}{2 h \nu} I^{2}(t, z)-\frac{N(t, z)}{\tau},
\end{gathered}
$$

where $\beta_{\text {TPA }}$ is the TPA coefficient, $\sigma$ the FCA coefficient, and $\tau$ the carrier lifetime. The equations are solved for a secant-hyperbolic pulse of $650 \mathrm{fs}$ (revealed from FROG measurements) assuming that the pulse train is sufficiently spaced in time thereby neglecting the effects of adjacent pulses on the free carrier density. This assumption follows from the carrier lifetime of a-Si:H which has been estimated to be as low as $\sim 400 \mathrm{ps}$ [6], considerably shorter than our repetition rate of $25 \mathrm{~ns}$. Equations (1) and (2) are used to fit the TPA and FCA coefficients to experimentally measured transmission powers. These values are then used in a modified form of the nonlinear Schrödinger equation (NLSE) evaluated with Eq. (3) and compared to the experimental spectra to determine the Kerr nonlinear refractive index $n_{2}$ [7]:

$$
\frac{\partial A(t, z)}{\partial z}=-\frac{i \beta_{2}}{2} \frac{\partial^{2} A(t, z)}{\partial t^{2}}+i \gamma|A(t, z)|^{2} A(t, z)-\frac{1}{2}\left(\sigma_{f}+\alpha_{l}\right) A(t, z)
$$

where $A(t, z)$ is the pulse envelope, the complex nonlinear parameter is $\gamma=\left(k_{0} n_{2}+i \beta_{\mathrm{TPA}} / 2\right) / A_{\mathrm{eff}}$, the free carrier contribution is $\sigma_{f}=\sigma(1+i \mu) N(t, z)$ and $\mu$ governs the free-carrier dispersion (FCD). In our experiments, the effects of GVD are negligible as the dispersion length is much greater than the length of the fiber used in this study.

Simulated absorption and spectral measurements illustrated in Fig. 1(a) and 1(b) reveal the TPA coefficient $\beta_{\text {TPA }} \sim$ $0.8 \mathrm{~cm} / \mathrm{GW}$, the FCA coefficient $\sigma \sim 1 \times 10^{-16} \mathrm{~cm}^{2}$ and $n_{2} \sim 1.8 \times 10^{-13} \mathrm{~cm}^{2} / \mathrm{W}$. The TPA parameter is within the reported range for that of crystalline silicon [8] and the FCA coefficient agrees with the Drude model as expected. In addition, we observe that the nonlinear refractive index is at least twice the $n_{2}$ of crystalline silicon [8]. Characterization of the aforementioned parameters provide insight to the potential use of these waveguides through the nonlinear figure of merit FOM $=2 \lambda \beta_{\text {TPA }} / n_{2}$. An FOM $<1$ implies a $4 \pi$ phase shift and FOM $<4$ produces a $\pi$ phase shift. The established quantities reveal an FOM $\sim 1.54$ inferring applications as nonlinear switching devices [9].

Lastly, we demonstrate an all-optical modulator based on degenerate two photon absorption. A pulsed pump wave at $1540 \mathrm{~nm}$ is launched in the presence of a CW probe at $1570 \mathrm{~nm}$, two photon absorption simultaneously occurs in the silicon fiber on the pump and probe, causing power depletion in the probe during the peak of a pump. Thus, the pump pulse sequence is inversely imprinted on the CW probe. Fig. 2(a) shows the result of the imprinted pulse on a $30 \mathrm{GHz}$ photodetector, the pump pulse is also illustrated to highlight the inverted nature of the probe. An expanded time-scale as in Fig. 2(b) emphasizes characteristics of the carrier recovery. An initial extinction ratio is estimated to 


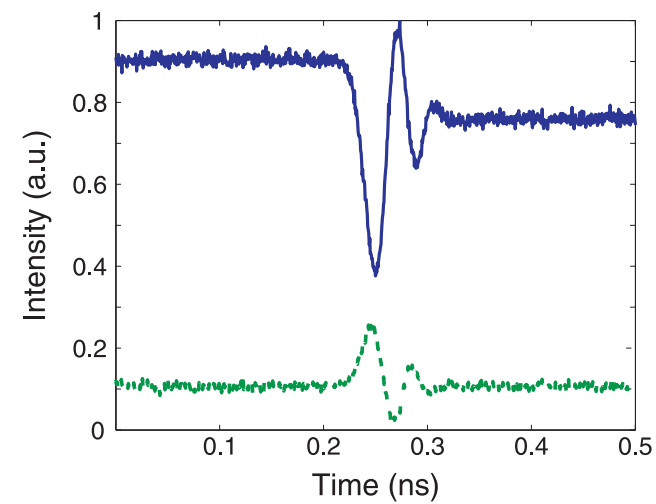

(a)

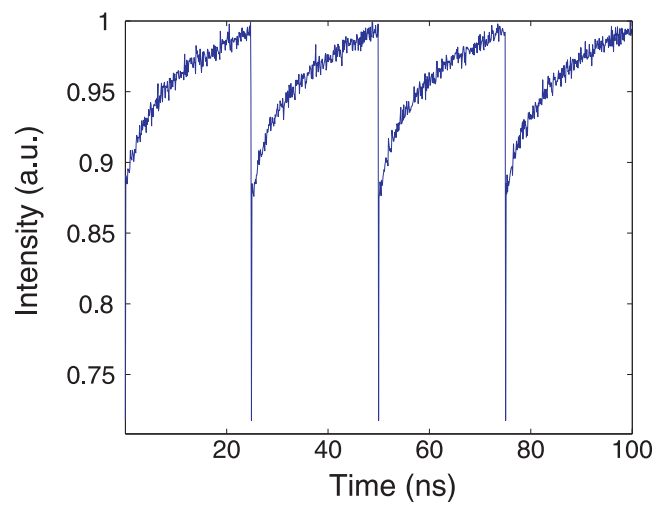

(b)

Fig. 2. (a) Dark probe pulse (solid line) and bright pump pulse (dashed line) from a $30 \mathrm{GHz}$ photodetector. (b) Train of dark pulses spaced by $25 \mathrm{~ns}$ corresponding to the pump's repetition rate

be $\sim 3 \mathrm{~dB}$. However, the slow transition time ( $\sim 16 \mathrm{ps})$ of our detector dramatically impedes the pulse shape and thus other methods are required to establish the response time and depth more accurately. We are currently investigating a range of linear and nonlinear techniques to recover a more definitive description of the modulated dark pulse.

\section{Conclusion}

We have successfully characterized nonlinear pulse propagation in a semiconductor-core optical fiber, revealing parameters in agreement with on-chip waveguides. Additionally, the first nonlinear optical device has been demonstrated in these fibers suggesting potential for future compact photonic applications.

\section{References}

1. K. Narayanan and S. F. Preble, "Optical nonlinearities in hydrogenated amorphous silicon waveguides," Opt. Express 18, 8998-9005 (2010).

2. P. J. A. Sazio, A. Amezcua-Correa, C. E. Finlayson, J. R. Hayes, T. J. Scheidemantel, N. F. Baril, B. R. Jackson, D. J. Won, F. Zhang, E. R. Margine, V. Gopalan, V. H. Crespi, and J. V. Badding, "Microstructured optical fibers as high-pressure microfluidic reactors," Science 311, 1583-1586 (2006).

3. P. Mehta, N. Healy, N. F. Baril, P. J. A. Sazio, J. V. Badding, and A. C. Peacock, "Nonlinear transmission properties of hydrogenated amorphous silicon core optical fibers," Opt. Express 16, 16826-16831 (2010).

4. L. Lagonigro, N. Healy, J. R. Sparks, N. F. Baril, P. J. A. Sazio, J. V. Badding, and A. C. Peacock, "Low loss silicon fibers for photonics applications," Appl. Phys. Lett. 96, 041105 (2010).

5. R. Dekker, N. Usechak, M. Forst, and A. Driessen, "Ultrafast nonlinear all-optical processes in silicon-on insulator waveguides," J.Phys D, Appl. Phys. 40, R249-R271 (2007).

6. K. Narayanan, A. W. Elshaari, and S. F. Preble, "Broadband all-optical modulation in hydrogenated-amorphous silicon waveguides," Opt. Express 18, 9809-9814 (2010).

7. L. Yin, and G. P. Agrawal, "Impact of two-photon absorption on self-phase modulation in silicon waveguides," Opt. Lett. 32, 2031-2033 (2007).

8. G. W. Rieger, K. S. Virk, and J. F. Young, "Nonlinear propagation of ultrafast $1.5 \mu \mathrm{m}$ pulses in high-index contrast silicon-on-insulator waveguides," Appl. Phys. Lett. 84, 900-902 (2004).

9. K.W. DeLong, K. B. Rochford, and G. I. Stegeman, "Effect of two-photon absorption on all-optical guided-wave devices," Appl. Phys. Lett. 55, 1823-1825 (1989). 